\title{
Characterization of Legionella pneumophila isolates from patients in Japan according to serogroups, monoclonal antibody subgroups and sequence types
}

\section{Correspondence Junko Amemura-Maekawa jmaekawa@nih.go.jp}

Received 11 November 2009 Accepted 21 February 2010
Junko Amemura-Maekawa, ${ }^{1}$ Fumiaki Kura, ${ }^{1}$ Jürgen $\mathrm{H}$. Helbig, ${ }^{2}$ Bin Chang, ${ }^{1}$ Akiko Kaneko, ${ }^{3}$ Yuko Watanabe, ${ }^{4}$ Junko Isobe, ${ }^{5}$ Masafumi Nukina, ${ }^{6}$ Hiroshi Nakajima, ${ }^{7}$ Kimiko Kawano, ${ }^{8}$ Yuki Tada, ${ }^{9}$ Haruo Watanabe ${ }^{1}$ and the Working Group for Legionella in Japant

\author{
${ }^{1}$ Department of Bacteriology I, National Institute of Infectious Diseases, Tokyo, Japan \\ ${ }^{2}$ Institute of Medical Microbiology and Hygiene, TU Dresden, Dresden, Germany \\ ${ }^{3}$ Yamagata Prefectural Institute of Public Health, Yamagata, Japan \\ ${ }^{4}$ Kanagawa Prefectural Institute of Public Health, Kanagawa, Japan \\ ${ }^{5}$ Toyama Institute of Health, Toyama, Japan \\ ${ }^{6}$ Public Health Research Institute of Kobe City, Hyogo, Japan \\ ${ }^{7}$ Okayama Prefectural Institute for Environmental Science and Public Health, Okayama, Japan \\ ${ }^{8}$ Miyazaki Prefectural Institute for Public Health and Environment, Miyazaki, Japan \\ ${ }^{9}$ Infectious Disease Surveillance Center, National Institute of Infectious Diseases, Tokyo, Japan
}

We collected 86 unrelated clinical Legionella pneumophila strains that were isolated in Japan during the period 1980-2008. Most (80.2\%) belonged to serogroup 1, followed by serogroups 5,3 and 2. Interestingly, the patients with $L$. pneumophila serogroup 1 had a significantly higher male-to-female ratio (12.4) than the patients with other $L$. pneumophila serogroups (2.0) (OR, $10.5 ; 95 \% \mathrm{Cl}, 2.5-44.5)$. When the serogroup 1 strains were analysed by monoclonal antibody (mAb) typing, the most prevalent subgroup was Benidorm (34.9\% of all isolates). Moreover, $79.7 \%$ of the serogroup 1 isolates were bound by $m A b 3 / 1$, which recognizes the virulenceassociated epitope. When all 86 isolates were subjected to sequence-based typing (SBT) using seven loci, they could be divided into 53 sequence types (STs). The ST with the most isolates (seven) was ST1, to which most isolates from patients and environments around the world belong. However, six of the seven ST1 isolates were isolated before 1994. Other major STs were ST306 $(n=6)$, ST120 $(n=5)$ and ST138 $(n=5)$. All ST306 and ST138 isolates, except for one isolate (ST306), were suspected or confirmed to be derived from bath water, which suggests that these strains prefer bath habitats. The sources of all ST1 and ST120 isolates remain unclear. By combining the SBT and mAb data, the 86 isolates could be divided into 59 types (discrimination index, 0.984). This confirms the usefulness of this combination in epidemiological studies.
†The members of the Working Group for Legionella in Japan are listed in Acknowledgements.

Abbreviations: EWGLI, European Working Group on Legionella Infections; SBT, sequence-based typing; ST, sequence type.

A supplementary table showing the sequence types, serogroups and monoclonal antibody subtypes of Japanese clinical isolates of Legionella pneumophila is available with the online version of this paper.

\section{INTRODUCTION}

Legionellosis is caused by Legionella species, which are environmental Gram-negative bacteria. To date, 52 species of Legionella have been described (Kuroki et al., 2007). The species that is most commonly found in the environment and also causes the most disease is Legionella pneumophila (Yu et al., 2002). To aid the epidemiological surveillance of legionellosis, L. pneumophila isolates can be divided into serogroups by antisera that recognize differences on the 
LPS molecules. At present, 15 serogroups have been identified (Brenner et al., 1988). Serogroup 1 is responsible for the majority of human infections (Yu et al., 2002) and its subgroups can be delineated by six monoclonal antibodies (mAbs) that recognize specific epitopes (Helbig et al., 1997). L. pneumophila isolates can also be characterized by sequence-based typing (SBT) using the six loci (flaA, pilE, asd, mip, mompS and proA) proposed by the European Working Group on Legionella Infections (EWGLI; http://www.ewgli.org/) (Gaia et al., 2005). Recently, to enhance the delineation of L. pneumophila strains, a seventh allele, neuA, has been added in SBT (Ratzow et al., 2007).

In Japan, National Epidemiological Surveillance of Infectious Diseases data indicate that hot springs and public baths but not cooling towers are the major sources of Legionella infections (Infectious Disease Surveillance Center, 2000). Indeed, there have been four large outbreaks in public bath facilities (Kuroki et al., 2009).

In our previous study (Amemura-Maekawa et al., 2005), we analysed 27 epidemiologically unrelated L. pneumophila serogroup 1 isolates (ten from cooling towers, ten from public spas and/or hot spring baths, and seven from patients with public bath-related infections) from Japan by SBT using the six alleles proposed in 2005 by the EWGLI. The 27 isolates could be divided into 14 sequence types (STs). Notably, the 10 isolates from the cooling towers all showed the same allele types, namely flaA (1), pilE (4), asd (3), mip (1), mompS (1) and proA(1), whereas the public bath-derived isolates were more diverse.

Here, to further characterize clinical L. pneumophila isolates from Japan and to confirm the usefulness of the $\mathrm{mAb}$ - and SBT-based classification methods, we analysed 86 isolates by both typing methods.

\section{METHODS}

L. pneumophila strains. We analysed 86 clinical isolates of $L$. pneumophila that were isolated in Japan during the period 1980-2008. Of these, 42 clinical Legionella isolates were from the Legionella Reference Center, which collects Legionella isolates obtained in six representative prefectural/municipal public health institutes of each district in Japan. The remaining 44 isolates were from the collection of the National Institute of Infectious Diseases, Department of Bacteriology I (NIIB). Of the 86 isolates, 84 were from unrelated cases. The remaining two isolates were obtained from the same patient but belonged to different serogroups (NIIB 2136 and NIIB 2137; Supplementary Table S1 in JMM Online). Nosocomial cases were defined as those in patients who had been hospitalized, and travel-associated cases were defined as those in patients who had spent at least one night away from home before onset of the symptoms. The incubation period was set to 2-10 days, but this depended on the discretion of the physician who notified a patient with legionellosis. Because previous studies (Infectious Disease Surveillance Center, 2000; Kuroki et al., 2009) have indicated that the major sources of Legionella infections are public baths without taking into consideration whether or not the infected individuals had engaged in previous travelling or not, the relationship between travelling and legionellosis has not been under active consideration.
Outbreaks were defined as two or more cases for which there was strong epidemiological evidence of a common source of infection, with or without microbiological evidence. The 86 isolates included one representative isolate from each of the 12 Legionella outbreaks; all of the outbreaks were community-acquired, of which four were major outbreaks (Kuroki et al., 2009).

Serogrouping and $\mathbf{m A b}$ subgrouping. Serogrouping of the isolates was performed by slide agglutination tests using a monovalent serum for L. pneumophila serogroups 1-15 (Denka Seiken). Serogroup 1 and 5 isolates were then subtyped serologically by using mAbs as described previously (Helbig et al., 1997).

DNA manipulation, sequencing and sequence typing. Genomic DNA was extracted by using a High Pure PCR Template Preparation kit (Roche Diagnostics) and amplified by using the GeneAmp PCR System 9700 (Applied Biosystems) and previously described reaction mixtures and conditions (Amemura-Maekawa et al., 2005; Gaia et al., 2005). Both strands of the amplicons were sequenced by a model 3100,3130 or $3130 x \mathrm{x}$ ABI Sequencer (Applied Biosystems). The nucleotide sequences obtained were confirmed and the allele numbers were determined using the online Sequence Quality Tool of the EWGLI website (Underwood et al., 2006). Putative novel variants were submitted to the site 'Sequence Quality Tool' for verification and assignment of new allelic numbers. New combinations of allelic numbers were also submitted to the curators via the EWGLI website for assignment of new ST numbers. In this study, the isolates that failed amplification of neuA (indicated allele number as ' 0 ') were not given ST numbers but were allocated arbitrary numbers that start with $\mathrm{J}(\mathrm{J} 1, \mathrm{~J} 2$, etc.). A minimum spanning tree was generated by BioNumerics software (version 5.1; Applied Maths) using as parameters the categorical coefficient of similarity and the priority rule of the highest number of single-locus variants.

\section{RESULTS AND DISCUSSION}

\section{Patient age, gender and sources of isolates}

The mean and median age of the 85 legionellosis patients (72 males, 10 females and 3 unknown cases) was 59.2 and 60 years, respectively (range $0-81$; excluding the three patients whose ages were unknown). It has been shown previously that many more males than females contract legionellosis (Infectious Disease Surveillance Center, 2008; Neil \& Berkelman, 2008; Ng et al., 2008), although the reasons for this are unclear. There were seven nosocomial cases (8.2\%), eight travel-associated cases (9.4\%), 49 community-acquired cases $(57.6 \%)$ and 21 cases for which the source could not be identified $(24.7 \%)$. The source of infection for 35 of the 85 patients was suspected to be public bathing facilities $(n=30,35.3 \%)$, many of which apparently had circulation systems and 25 of which used hot-spring waters, domestic baths $(n=2)$, a shower $(n=1)$, a humidifier $(n=1)$ and a cooling tower $(n=1)$. The sources of the infection for 16 of these 35 cases were indeed confirmed to be public baths $(n=15)$ and a humidifier $(n=1)$ by comparing the PFGE DNA patterns (AmemuraMaekawa et al., 2005) of the clinical isolates to those of environmental isolates from the suspected origin of the infection at prefectural/municipal public health institutes. The sources of infection of the remaining 50 cases $(61.2 \%)$ are unknown. In addition, 12 isolates $(14.1 \%)$ were 
derived from 12 individual outbreaks, of which 9, 1, 1 and 1 outbreaks occurred at public bath facilities (hot-spring waters were used at eight of the nine facilities and tap water was used at the remaining one), a nursing home (Maesaki et al., 1992), the cooling tower of a waste-processing plant (Isozumi et al., 2005) and the spa of a cruise ship (Kura et al., 2006) (Supplementary Table S1), respectively.

\section{Serogroups}

The majority of the isolates $(n=69,80.2 \%)$ belonged to serogroup 1 , including 11 of 12 outbreak-derived isolates. Two, five, one, seven (including the remaining one outbreak-derived isolate), one and one isolate(s) belonged to serogoups 2, 3, 4, 5, 6 and 9, respectively (Supplementary Table S1). The majority of the male patients $(87.5 \%)$ were infected with serogroup 1 strains whereas only $40 \%$ of the female patients were infected with serogroup 1 strains. Thus, the serogroup 1-infected patients were significantly more likely to be male (male: female ratio $=12.4$ ) than the patients infected with isolates belonging to other serogroups (male:female ratio $=2.0$; OR, $10.5 ; 95 \% \mathrm{CI}, 2.5-44.5$ ). The reasons for this difference remain to be elucidated.

\section{mAb subgroups}

Of the 69 serogroup 1 isolates, $79.7 \%(n=55)$ had the virulence-associated epitope that is recognized by $\mathrm{mAb} 3 /$ 1 and is not present on any other serogroups (Helbig et al., 2002). Thus, of the 86 Legionella isolates, $64.0 \%$ were $\mathrm{mAb} 3 / 1$-positive, which is similar to the frequency of $66.8 \%$ reported by the pan-European study. The 69 serogroup 1 isolates belonged to the Benidorm (44.9\%), Allentown/France (17.4\%), OLDA (14.5\%), Philadelphia (10.1\%), Knoxville (7.2\%), Oxford (4.4\%) and Bellingham $(1.5 \%) \mathrm{mAb}$ subgroups. The distribution of $\mathrm{mAb}$ subgroups in Japanese isolates differs from that in pan-European isolates, which most frequently have the Philadelphia subgroup (28.5\%) and then the Benidorm subgroup (20.0\%) (Helbig et al., 2002). Eleven of the 12 outbreak-derived isolates were mAb 3/1-positive (the single serogroup 5 outbreak-derived isolate lacked this marker).

\section{STs}

The 86 clinical isolates could be divided into 53 STs (discrimination index, 0.979) (Hunter \& Gaston, 1988), though amplification of the neuA target failed for eight isolates of serogroups 2, 4 and 5 (Table 1 and Supplementary Table S1). The minimum spanning tree illustrates the distribution of the STs (Fig. 1). The tree has seven clonal complexes. The ST with the largest number of isolates was ST1 (seven isolates). ST1 is the most prevalent ST in the world (Borchardt et al., 2008; Cazalet et al., 2008; Harrison et al., 2009; Kozak et al., 2009; Reimer et al., 2010), although a Canadian study has reported that the prevalence of ST1 clinical isolates has decreased dramatically during the past 12 years (Tijet et al., 2010). Indeed, in our study, six of the seven known clinical ST1 strains were isolated before 1994, and it is now unusual to isolate clinical ST1 strains; thus, it is striking that the majority of environmental isolates from cooling tower water still belong to ST1 (Amemura-Maekawa et al., 2005; Cazalet et al., 2008). The next major STs were ST306 (six isolates), ST120 (five isolates) and ST138 (five isolates). Notably, all of the ST138 and ST306 isolates but one (ST306) were suspected or confirmed to be derived from public bath water, which suggests that these strains prefer bath habitats. Both ST138 and ST306 are unique to Japan according to data submitted to the EWGLI SBT database as of 12 January 2010. In contrast, the sources of all but one of the Japanese isolates belonging to ST1 and ST120 remain unclear (one isolate was suspected to come from shower water). Four isolates were ST23 strains, two of which (Supplementary Table S1, NIIB 292 and NIIB 374) were derived from two large public bath facility-associated outbreaks that affected hundreds of people (Nakamura et al., 2003; Okada et al., 2005). In Europe, clinical isolates often belong to ST23 (Borchardt et al., 2008; Cazalet et al., 2008). These major STs (ST1, ST23, ST120 and ST306), except for ST138, belonged to clonal complexes, and these STs were distinct from each other, with the exception of ST23 and ST120 (Fig. 1). ST430 and J2 had three isolates each, and the other seven STs had two isolates each (Table 1). The remaining 39 STs each had one isolate. Thirty-four of the 53 STs were unique to Japan as of 12 January 2010, including two SBT profiles that could not be assigned to STs because they failed neuA amplification (Table 1). With regard to the 12 outbreakderived strains, two belonged to each of ST23, ST138 and ST139, and the remaining six belonged to different STs (ST2, ST36, ST89, ST142, ST434 and J4). Notably, for ST142, there was not only an outbreak-derived isolate but also another unrelated non-outbreak-derived isolate. Similarly, ST2, ST36 and ST89 isolates have also been found in patients elsewhere in Japan (unpublished results) and/or abroad, as Philadelphia 1 was typed to ST36 (Ratzow et al., 2007).

The discrimination index (0.979) in our investigation was higher than that described in previous reports which were based on isolates from England and Wales (0.901; Harrison et al., 2009), USA (0.946; Kozak et al., 2009) and Canada (0.964; Reimer et al., 2010). This may reflect the different infection sources in Japan: the main infection source was public bath water (Supplementary Table S1), whereas those of outbreaks in Europe were hot- or coldwater systems and cooling towers (Ricketts et al., 2007). (There have been no reports for the dataset of the infection sources of sporadic cases in Europe, as far as we know.) The water of Japanese public baths is often obtained from hot springs. The characteristics of hot spring water, namely chemical features such as $\mathrm{pH}$ and temperature, are highly variable, whereas the water from hot- or cold-water systems and cooling towers tends to 
Table 1. Sequence types of 86 Japanese clinical isolates of Legionella pneumophila

ST, Sequence type: seven-allele profile; J2 and J5, arbitrary numbers allocated to each unique six-allele profile without neuA; Others, STs each presented by only a single isolate.

\begin{tabular}{|lccl|}
\hline ST & No. of isolates & $\%$ & mAb (no. of isolates) \\
\hline Serogroup 1 & 69 & 80.2 & \\
1 & 7 & 8.1 & OLDA (6), Oxford (1) \\
$306^{*}$ & 6 & 7.0 & Benidorm \\
$138^{*}$ & 5 & 5.8 & Benidorm (4), Allentown/France (1) \\
120 & 5 & 5.8 & Benidorm \\
23 & 4 & 4.7 & Allentown/France (2), Philadelphia (1), Oxford (1) \\
$122^{*}$ & 2 & 2.3 & Benidorm \\
42 & 2 & 2.3 & Benidorm \\
$118^{*}$ & 2 & 2.3 & Philadelphia \\
$123^{*}$ & 2 & 2.3 & Benidorm \\
$139^{*}$ & 2 & 2.3 & Allentown/France (1), Benidorm (1) \\
$142^{*}$ & 2 & 2.3 & Allentown/France \\
Others $\dagger$ & 30 & 34.9 & (Not shown) \\
Other serogroups (serogroup) & 17 & 19.8 & \\
$430^{*}(3)$ & 3 & 3.5 & \\
J2 $(5)$ & 3 & 3.5 & Dallas (2), Cambridge (1) \\
J5 (5) & 2 & 2.3 & Dallas \\
Others $¥(2,3,4,5,6,9)$ & 9 & (Not shown) \\
Total & 86 & 10.5 & \\
\hline
\end{tabular}

${ }^{\star}$ Described only in Japan as of 12 January 2010.

$\dagger$ Nineteen of 30 STs only in Japan as of 12 January 2010.

$\ddagger$ Seven of nine STs were described only in Japan as of 12 January 2010 .

have rather similar characteristics due to similar water treatment procedures in addition to the environmental selective pressure. Thus, reflecting the wider array of Legionella-suitable environmental niches in Japan, the Japanese clinical isolates may be more genetically variable than Western isolates. This idea is supported by previous reports from our laboratory, which showed that Japanese isolates derived from public baths differ genetically from Japanese isolates derived from cooling towers (AmemuraMaekawa et al., 2005, 2008).

\section{Combining sequence typing and mAb subgrouping}

Some STs were composed of isolates belonging to the different $m A b$ subgroups (and vice versa). Thus, six of the seven ST1 isolates were OLDA subgroup $(n=6)$ and the remaining ST1 isolate was an Oxford subgroup, while two of the four ST23 isolates were Allentown/France subgroup and the other two were Philadelphia and Oxford subgroups. All six ST306, all five ST120 and four of the five ST138 isolates were Benidorm subgroup. Moreover, in six of the seven STs, each of which consisted of two isolates, both isolates belonged to the same subgroup. By combining the SBT and subgrouping data, we could divide the 86 isolates into 59 types (discrimination index, 0.984) (Table 1 and Supplementary Table S1).

\section{Importance of Legionella isolation}

Based on the Infectious Diseases Control Law, legionellosis in Japan has been classified as a category IV notifiable infectious disease and has been monitored by the National Epidemiological Surveillance of Infectious Diseases (NESID) since 1999. Consequently, in Japan, physicians must notify the authorities about legionellosis cases. In 2008, 884 legionellosis cases were reported, which represents a remarkable fivefold increase in the previous 5 years (Infectious Disease Surveillance Center, 2008). However, this increase is thought to be due to the widespread use of a highly accurate urine antigen assay, which is easier to perform than the laborious and time-consuming process of isolating Legionella from the patients. From January 2003 to September 2008, 2460 legionellosis cases were reported, of which only 97 cases were diagnosed by the isolation of Legionella (Infectious Disease Surveillance Center, 2008). We collected 29 isolates during the same period. Therefore, our study may not entirely faithfully delineate the strains responsible for legionellosis in Japan. Health workers should isolate the bacterium from the patient and identify the infection source by genotyping the organism and comparing this genotype to those of environmental isolates from the surroundings of the patient.

The legionellosis incidence in Japan did not vary in a seasonal fashion (Infectious Disease Surveillance Center, 


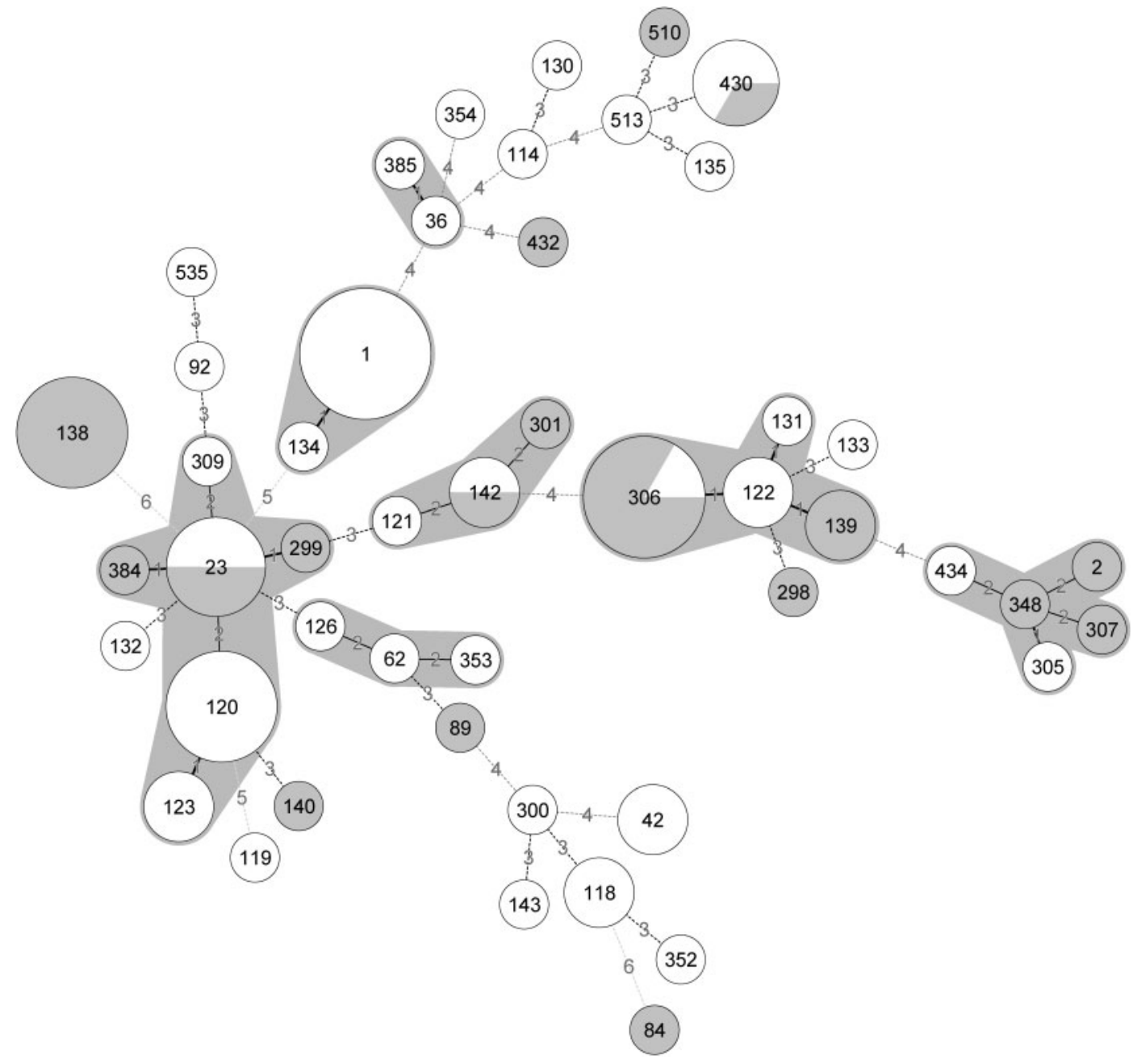

Fig. 1. Minimum spanning tree showing how the L. pneumophila clinical isolates, with seven determined alleles, are distributed in terms of their STs. The ST number is shown in the circle and the size of the circle indicates the number of isolates. Short thick lines connect single-locus variants, thin lines connect double-locus variants, black broken lines connect triple-locus variants and grey broken lines connect more than three different STs. The grey parts of the circles (pie charts) indicate the clinical isolates associated with bath water. The clonal complexes that were generated with single-locus variants and double-locus variants are indicated by the shaded backgrounds.

2003) until 2005, which is when the number of legionellosis cases started peaking in July (Infectious Disease Surveillance Center, 2008). This seasonality relates to humidity: rainfall increases microbial contamination in source waters or water distribution systems (Fisman et al., 2005; Ng et al., 2008). However, the epidemiological profile of sporadic legionellosis remains poorly understood. It has recently been proposed that legionellosis can be acquired from puddles on asphalt roads on rainy days (Sakamoto et al., 2009a) and from the air-conditioning systems of motor cars (Sakamoto et al., 2009b). It is likely that there are many as yet unrecognized infection sources of Legionella. It is possible that groups of isolates with particular genotypes inhabit distinct infection sources including unrecognized sources.

\section{ACKNOWLEDGEMENTS}

We thank Dr Norman K. Fry (Respiratory and Systemic Infection Laboratory, Health Protection Agency, UK) for assigning the newly identified alleles and STs. We are also grateful to the municipal and prefectural public health institutes, the public health centres, the infectious disease surveillance centres and the medical institutions in Japan that provided isolates and valuable information about the cases. This work was supported by Health and Labour Sciences Research Grants (H18-kenki-012 and H19-kenki-014 to F. K.).

The following are the members of the Working Group for Legionella in Japan: K. Ohtani and T. Aoki, Yamagata Prefectural Institute of Public Health; K. Mashiko, Ibaraki Prefectural Institute of Public Health; T. Kurazono, M. Kon and N. Shimada, Saitama Institute of Public Health; K. Tateda, Department of Microbiology and Infectious Diseases, Toho University Faculty of Medicine; H. Eguchi, Niigata 
City Institute of Public Health and Environment; T. Koyama, Nagano Environmental Conservation Research Institute; K. Sugiyama, Shizuoka Institute of Environment and Hygiene; Y. Kanazawa, Shizuoka City Institute of Environmental Sciences and Public Health; N. Furuta, Gifu Prefectural Research Institute for Health and Environmental Sciences; N. Agata, Nagoya City Public Health Research Institute; T. Kobayashi, Respiratory Division, Internal Medicine, Ichinomiyanishi Hospital; Y. Ito, Department of Respiratory Medicine, Graduate School of Medicine, Kyoto University; M. Taguchi, Osaka Prefectural Institute of Public Health; H. Tsuji, Hyogo Prefectural Institute of Public Health and Environmental Sciences; K. Ogata, Oita Prefectural Institute of Health and Environment; K. Horikawa, Fukuoka Institute of Health and Environmental Sciences; K. Uryu, Fukuoka City Institute for Hygiene and the Environment; Y. Shimizu, Kitakyushu City Institute of Environmental Sciences; A. Saito, Japanese Red Cross Nagasaki Genbaku Isahaya Hospital; N. Ueno and S. Kubozono, Kagoshima Prefectural Institute of Environmental Research and Public Health.

\section{REFERENCES}

Amemura-Maekawa, J., Kura, F., Chang, B. \& Watanabe, H. (2005). Legionella pneumophila serogroup 1 isolates from cooling towers in Japan form a distinct genetic cluster. Microbiol Immunol 49, 10271033.

Amemura-Maekawa, J., Kura, F., Chang, B., Suzuki-Hashimoto, A., Ichinose, M., Endo, T. \& Watanabe, H. (2008). Distinct difference of flaA genotypes of Legionella pneumophila between isolates from bath water and cooling tower water. Microbiol Immunol 52, 460-464.

Borchardt, J., Helbig, J. H. \& Lück, P. C. (2008). Occurrence and distribution of sequence types among Legionella pneumophila strains isolated from patients in Germany: common features and differences to other regions of the world. Eur J Clin Microbiol Infect Dis 27, 2936.

Brenner, D. J., Steigerwalt, A. G., Epple, P., Bibb, W. F., McKinney, R. M., Starnes, R. W., Colville, J. M., Selander, R. K., Edelstein, P. H. \& Moss, C. W. (1988). Legionella pneumophila serogroup Lansing 3 isolated from a patient with fatal pneumonia, and descriptions of $L$. pneumophila subsp. pneumophila subsp. nov., L. pneumophila subsp. fraseri subsp. nov., and L. pneumophila subsp. pascullei subsp. nov. J Clin Microbiol 26, 1695-1703.

Cazalet, C., Jarraud, S., Ghavi-Helm, Y., Kunst, F., Glaser, P., Etienne, J. \& Buchrieser, C. (2008). Multigenome analysis identifies a worldwide distributed epidemic Legionella pneumophila clone that emerged within a highly diverse species. Genome Res 18, 431-441.

Fisman, D. N., Lim, S., Wellenius, G. A., Johnson, C., Britz, P., Gaskins, M., Maher, J., Mittleman, M. A., Spain, C. V. \& other authors (2005). It's not the heat, it's the humidity: wet weather increases legionellosis risk in the greater Philadelphia metropolitan area. J Infect Dis 192, 2066-2073.

Gaia, V., Fry, N. K., Afshar, B., Lück, P. C., Meugnier, H., Etienne, J., Peduzzi, R. \& Harrison, T. G. (2005). Consensus sequence-based scheme for epidemiological typing of clinical and environmental isolates of Legionella pneumophila. J Clin Microbiol 43, 2047-2052.

Harrison, T. G., Afshar, B., Doshi, N., Fry, N. K. \& Lee, J. V. (2009). Distribution of Legionella pneumophila serogroups, monoclonal antibody subgroups and DNA sequence types in recent clinical and environmental isolates from England and Wales (2000-2008). Eur J Clin Microbiol Infect Dis 28, 781-791.

Helbig, J. H., Kurtz, J. B., Pastoris, M. C., Pelaz, C. \& Lück, P. C. (1997). Antigenic lipopolysaccharide components of Legionella pneumophila recognized by monoclonal antibodies: possibilities and limitations for division of the species into serogroups. J Clin Microbiol 35, 2841-2845.

Helbig, J. H., Bernander, S., Castellani Pastoris, M., Etienne, J., Gaia, V., Lauwers, S., Lindsay, D., Lück, P. C., Marques, T. \& other authors (2002). Pan-European study on culture-proven Legionnaires' disease: distribution of Legionella pneumophila serogroups and monoclonal subgroups. Eur J Clin Microbiol Infect Dis 21, 710-716.

Hunter, P. R. \& Gaston, M. A. (1988). Numerical index of the discriminatory ability of typing systems: an application of Simpson's index of diversity. J Clin Microbiol 26, 2465-2466.

Infectious Disease Surveillance Center (2000). Legionellosis, April 1999-July 2000. IASR 21, 186-187.

Infectious Disease Surveillance Center (2003). Legionellosis, April 1999-December 2002, Japan. IASR 24, 27-28.

Infectious Disease Surveillance Center (2008). Legionellosis, January 2003-September 2008, Japan. IASR 29, 327-328.

Isozumi, R., Ito, Y., Ito, I., Osawa, M., Hirai, T., Takakura, S., linuma, Y., Ichiyama, S., Tateda, K. \& other authors (2005). An outbreak of Legionella pneumonia originating from a cooling tower. Scand J Infect Dis 37, 709-711.

Kozak, N. A., Benson, R. F., Brown, E., Alexander, N. T., Taylor, T. H., Jr, Shelton, B. G. \& Fields, B. S. (2009). Distribution of lag-1 alleles and sequence-based types among Legionella pneumophila serogroup 1 clinical and environmental isolates in the United States. J Clin Microbiol 47, 2525-2535.

Kura, F., Amemura-Maekawa, J., Yagita, K., Endo, T., Ikeno, M., Tsuji, H., Taguchi, M., Kobayashi, K., Ishii, E. \& Watanabe, H. (2006). Outbreak of Legionnaires' disease on a cruise ship linked to spa-bath filter stones contaminated with Legionella pneumophila serogroup 5. Epidemiol Infect 134, 385-391.

Kuroki, H., Miyamoto, H., Fukuda, K., lihara, H., Kawamura, Y., Ogawa, M., Wang, Y., Ezaki, T. \& Taniguchi, H. (2007). Legionella impletisoli sp. nov. and Legionella yabuuchiae sp. nov., isolated from soils contaminated with industrial wastes in Japan. Syst Appl Microbiol 30, 273-279.

Kuroki, T., Ishihara, T., Ito, K. \& Kura, F. (2009). Bathwater-associated cases of legionellosis in Japan, with a special focus on Legionella concentrations in water. Jpn J Infect Dis 62, 201-205.

Maesaki, S., Kohno, S., Koga, H., Kaku, M., Yoshitomi, Y., Yamada, H., Matsuda, H., Higashiyama, Y., Hara, K. \& other authors (1992). An outbreak of Legionnaires' pneumonia in a nursing home. Intern Med 31, 508-512.

Nakamura, H., Yagyu, H., Kishi, K., Tsuchida, F., Oh-Ishi, S., Yamaguchi, K. \& Matsuoka, T. (2003). A large outbreak of Legionnaires' disease due to an inadequate circulating and filtration system for bath water - epidemiologic manifestations. Intern Med 42, 806-811.

Neil, K. \& Berkelman, R. (2008). Increasing incidence of legionellosis in the United States, 1990-2005: changing epidemiologic trends. Clin Infect Dis 47, 591-599.

Ng, V., Tang, P., Jamieson, F., Drews, S. J., Brown, S., Low, D. E., Johnson, C. C. \& Fisman, D. N. (2008). Going with the flow: legionellosis risk in Toronto, Canada is strongly associated with local watershed hydrology. EcoHealth 5, 482-490.

Okada, M., Kawano, K., Kura, F., Amemura-Maekawa, J., Watanabe, H., Yagita, K., Endo, T. \& Suzuki, S. (2005). The largest outbreak of legionellosis in Japan associated with spa baths: epidemic curve and environmental investigation. Kansenshogaku Zasshi 79, 365-374 (in Japanese).

Ratzow, S., Gaia, V., Helbig, J. H., Fry, N. K. \& Lück, P. C. (2007). Addition of neuA, the gene encoding $N$-acylneuraminate cytidylyl transferase, increases the discriminatory ability of the consensus 
sequence-based scheme for typing Legionella pneumophila serogroup 1 strains. J Clin Microbiol 45, 1965-1968.

Reimer, A. R., Au, S., Schindle, S. \& Bernard, K. A. (2010). Legionella pneumophila monoclonal antibody subgroups and DNA sequence types isolated in Canada between 1981 and 2009: Laboratory Component of National. Eur J Clin Microbiol Infect Dis 29, 191-205.

Ricketts, K. D., Joseph, C. A. \& European Working Group for Legionella Infections (2007). Legionnaires disease in Europe: 20052006. Euro Surveill 12, E7-E8.

Sakamoto, R., Ohno, A., Nakahara, T., Satomura, K., Iwanaga, S., Kouyama, Y., Kura, F., Kato, N., Matsubayashi, K. \& other authors (2009a). Is a puddle on asphalt roads on rainy days a risk for Legionnaires' disease? Emerg Infect Dis 15, 1295-1297.

Sakamoto, R., Ohno, A., Nakahara, T., Satomura, K., Iwanaga, S., Kouyama, Y., Kura, F., Noami, M., Kusaka, K. \& other authors (2009b). Is driving a car a risk for Legionnaires' disease? Epidemiol Infect 137, 1615-1622.
Tijet, N., Tang, P., Romilowych, M., Duncan, C., Ng, V., Fisman, D. N., Jamieson, F., Low, D. E. \& Guyard, C. (2010). New endemic Legionella pneumophila serogroup I clones, Ontario, Canada. Emerg Infect Dis 16, 447-454.

Underwood, A., Bellamy, W., Afshar, B., Fry, N. \& Harrison, T. (2006). Development of an online tool for the European Working Group for Legionella Infections sequence-based typing, including automatic quality assessment and data submission. In Legionella: State of the Art 30 Years After Its Recognition, pp. 163-166. Edited by N. P. Cianciotto, Y. Abu Kwaik, P. H. Edelstein, B. S. Fields, D. F. Geary, T. G. Harrison, C. A. Joseph, R. M. Ratcliff, J. E. Stout \& M. S. Swanson. Washington, DC: American Society for Microbiology.

Yu, V. L., Plouffe, J. F., Pastoris, M. C., Stout, J. E., Schousboe, M., Widmer, A., Summersgill, J., File, T., Heath, C. M. \& other authors (2002). Distribution of Legionella species and serogroups isolated by culture in patients with sporadic community-acquired legionellosis: an international collaborative survey. J Infect Dis 186, 127-128. 